\title{
Editorial
}

\section{De la atención de la enfermedad hacia la promoción de la salud, contruyendo un nuevo paradigma}

Albert Einstein decía que "el principio de la locura, es creer que haciendo lo mismo obtendremos resultados diferentes"; y esta frase puede ser particularmente cierta en salud. En los últimos años se ha observado, en la clínica, el resurgir de patologías que se creían controladas, de nuevo se atienden niños con parasitosis, con desnutrición, con tuberculosis cerebral; adultos jóvenes con patologías como cáncer, diabetes e infartos; ancianos que padecen enfermedades y situaciones derivadas de inadecuados estilos de vida. Nuestro entorno cada día es menos saludable, lo que repercute en violencia, drogadicción y estrés. Somos conscientes de la inequidad que en materia de salud ha dividido el país en dos clases de usuarios, los que asisten a servicios públicos y los que recurren a las facilidades privadas. Cada vez más enfrentamos el reto de la obesidad, el sedentarismo, el consumo de tabaco y todas las enfermedades que de ello se originan. Observamos con preocupación cómo se deteriora nuestra infraestructura hospitalaria y cómo no siempre los fondos se invierten con sentido común y social. Pero seguimos pensando que construyendo hospitales y clínicas, contando con el equipo diagnóstico, el tratamiento más sofisticado y los medicamentos de última línea solucionaremos todos los problemas de salud de la población. Lamentablemente, nos equivocamos...y por ello se debe modificar el modelo de salud del país.

Durante los próximos cuatro años el Ministerio de Salud trabajará en la construcción de un nuevo paradigma: promoción de la salud, más que atención de la enfermedad, no como un simple lema, sino como una necesidad real en la cual la promoción de la salud debe ser un eje transversal de todo acto médico y sanitario, por más complejo y sofisticado que sea. Pero para ello es necesario comprender lo que esto significa.

Históricamente, la forma como una sociedad aborda la salud de sus miembros está muy ligada con la manera en la cual conceptualiza el proceso salud-enfermedad, lo que condiciona la respuesta social a los problemas de salud individuales y colectivos.

A principios del siglo XX, según el concepto biologista predominante del proceso saludenfermedad, en las instituciones de salud se partía de que, para proteger y mejorar el nivel de salud de la población, debían desarrollarse programas de atención dirigidos a curar a las personas enfermas. De lo anterior se desprende que la hipótesis prevaleciente era que "cuantas más personas enfermas se curen, más sana es la población”.

Como consecuencia del desarrollo de las ciencias de la salud y de los cambios en el perfil epidemiológico de las poblaciones, el concepto de cómo se produce la salud evolucionó, y a partir de los años sesentas aparecieron los primeros pensadores con nuevos enfoques, quienes planteaban que la aparición de la enfermedad ocurre cuando una serie de factores nosológicos actúan sobre el organismo por un periodo determinado, en forma silenciosa, sobreviniendo luego la respuesta corporal y las manifestaciones de la enfermedad. Entre los eventos causales que se mencionaban se incluían factores biológicos, culturales, socioeconómicos, ambientales, entre otros.

Debido a esto, con más fuerza a partir de la segunda mitad del siglo pasado, se reconoció que existen grupos de la población expuestos a ciertos riesgos y que es posible, mediante algunas intervenciones, prevenir que las personas enfermen. Así, se comenzaron a organizar programas 
A partir de la década de los setenta se empezó a reconocer explícitamente el hecho de que el nivel de salud de una población no depende en exclusiva de lo que hacen las instituciones vinculadas a la salud, sino que este es el resultado del desarrollo armónico de la sociedad en su conjunto, tomando luego fuerza el concepto de producción social de la salud, que se entiende como el proceso mediante el cual la interacción entre los actores sociales y entre estos y su entorno, genera como resultado final el estado de salud que caracteriza a una población, definiéndose como actor social a todo individuo, colectividad u organización cuyo accionar tiene un efecto significativo sobre el proceso de producción de la salud, sea este positivo o negativo, incluida la toma de decisiones relacionada con la formulación de las políticas públicas en cualquier ámbito.

Un modelo explicativo de la manera como se produce el nivel salud en una población fue desarrollado en la década de 1970 por el gobierno canadiense, impulsado por el Dr. Marc Lalonde; este modelo anota que los factores que inciden en el proceso de producción social de la salud pueden ser agrupados en cuatro categorías:

- Factores biológicos, que se refieren a todos aquellos elementos de salud, tanto física como mental, desarrollados dentro del cuerpo humano como consecuencia de la biología básica y de aspectos orgánicos del individuo, por ejemplo: la carga genética, el proceso de envejecimiento, los sistemas internos del organismo y otros.

- Factores ambientales, incluyendo los relacionados con el hábitat humano (condiciones de saneamiento básico y de vivienda, el medio laboral, el escolar y otros).

- Factores socioeconómicos y culturales (estilos de vida, decisiones y hábitos, ingreso económico, nivel educativo, aspectos culturales, empleo, recreación, participación política).

- Factores relacionados con servicios de salud de atención a las personas (acceso, cantidad, calidad, oportunidad y organización de estos servicios de salud).

De lo mencionado se desprende que la salud, como producto social, depende de los diversos determinantes y de la respuesta social generada para abordarla, por lo que, sobre la base de este conocimiento, se empezó a enfatizar en que, además de realizar acciones preventivas dirigidas a grupos de riesgo, para proteger y mejorar el estado de salud de la población, se debe apoyar a quienes tengan la capacidad de adoptar estilos de vida saludables y participar activamente en el cuidado de su salud, la de su familia y la de su comunidad, y brindar las condiciones y requisitos para que esto tenga lugar, es decir, promocionar la salud. Fue entonces cuando, en prácticamente todos los países latinoamericanos, algunas instituciones de salud crearon y otras fortalecieron programas de educación y comunicación en salud y participación comunitaria, aunque no abordaron con la misma fuerza la realización de acciones dirigidas a modificar los determinantes del entorno.

Se sabe que el nivel de salud de los países desarrollados no se debe mayormente a un gasto per cápita en salud más alto, ni a la inversión en tecnologías de avanzada dedicadas al diagnóstico y tratamiento de personas enfermas; más bien se explica por la presencia de factores nutricionales $y$ ambientales más favorables para el ser humano. Existe evidencia de que en la variación del perfil de salud de una población, el impacto de los servicios de salud de atención a las personas no sobrepasa el $20 \%$, mientras que cerca del $80 \%$ restante es atribuible a la modificación de factores externos a estos servicios, razón por la cual hay que tener claro que la atención curativa impacta primordialmente sobre la mortalidad, pero tiene poco efecto sobre la morbilidad. Ante este conocimiento, resulta paradójico el hecho de que la gran mayoría de los esfuerzos y recursos estatales destinados a proteger y mejorar el estado de salud de la población se continúen invirtiendo en los servicios de salud, principalmente en aquellos dirigidos a la atención individual de las personas, y el enfoque predominante continúa siendo el de modificar los factores de riesgo, dejando de lado, en muchos casos, las acciones para propiciar el desarrollo de los factores protectores.

Durante los próximos cuatro años este será el modelo que el Ministerio de Salud seguirá, interactuando a través de alianzas estratégicas con todas las instituciones que conforman el sector salud. Es preciso recordar que si bien la atención directa de la enfermedad es muy importante y debe brindarse con criterios de calidad, de equidad y de oportunidad, el mejoramiento del hábitat humano permitirá tener un país y, por ende, una población más sana.

No es posible mejorar la salud sin manejar de manera adecuada los determinantes sociales de esta, lo cual se logrará mediante la estrategia de la promoción de la salud, utilizando la definición de la Carta de Ottawa "proporcionar a los pueblos los medios necesarios para mejorar su salud y ejercer un mayor control sobre la misma. Trasciende la idea de formas de vidas sanas para incluir las condiciones $y$ requisitos para la salud que son: paz, vivienda, educación, alimentación, renta, ecosistema estable, recursos sostenibles, justicia social y equidad". Constituye un proceso político y social global que abarca no solo las acciones orientadas a fortalecer las habilidades y capacidades de los individuos, sino también las dirigidas a modificar las condiciones sociales, ambientales y económicas, con el fin de mitigar su impacto en la salud pública e individual.

De la definición anterior se desprende que la promoción de la salud es una función que trasciende al Ministerio de Salud y que permite alcanzar mejoras significativas y equitativas en la condición sanitaria de la población, pues implica que los componentes del Sistema de Producción Social de la Salud, dirigidos y conducidos por el Estado, actúen de manera armónica para proporcionar las 
herramientas a los ciudadanos que les permitan cuidar su salud, y para brindarles las mejores opciones políticas, económicas, legales, ambientales, educativas, de recreación, de inclusión en la toma de decisiones, entre otras, con la finalidad de que desarrollen sus potencialidades en aras del bienestar individual y colectivo. No cabe duda entonces de que las instituciones del Estado deben realizar sus funciones, en concordancia con este enfoque, para contribuir al logro de un nivel óptimo de salud y de desarrollo humano.

El reto es grande y los resultados esperados son ambiciosos, pero el esfuerzo vale la pena. Dra. Rossana García-González
Directora General de Salud

Dra. María Luisa Ávila-Agüero Ministra de Salud

\section{Referencias}

1. The PLoS Medicine Editors; Stonington S, Holmes SM. Social Medicine in the Twenty-First Century. PLoS Med. 2006, 24;3

2. Porter D.How Did Social Medicine Evolve, and Where Is It Heading? PLoS Med. 2006, 24;3

3. de Castro-Santos LA.Public health policy and the building up of a Brazilian medical identity. Parassitologia. 2005;47:387-95.

4. Wiist WH .Public health and the anticorporate movement: rationale and recommendations. Am J Public Health. 2006;96:1370-5.

5. Berridge V.Social medicine: national and international transfer of ideas. J Epidemiol Community Health. 2006;60:558.

6. Blane D.Commentary: the place in life course research of validated measures of socioeconomic position. Int J Epidemiol. 2006;35:139-40.

7. Kunze M, Vutuc C.Health determinants in Europe for indoor and outdoor pollutants from a public health and social medicine view. Exp Toxicol Pathol. 2005;57:9-17.

8. Likic R, Dusek T, Horvat D.Analysis and prospects for curricular reform of medical schools in Southeast Europe. Med Educ. 2005;39:833-40.

9. Gramlich EM.Social security reform in the twenty-first century: the United States. J Aging Soc Policy. 2002;14:67-80.

10. Jordanova L.The social construction of medical knowledge. Soc Hist Med. 1995;8:361-81 